\title{
Racionalidade limitada e a tomada de decisão em sistemas complexos
}

\author{
Bounded rationality and decision-making in complex systems
}

\author{
TATIANA MASSAROLI MELO \\ JOSÉ RICARDO FUCIDJI*
}

\begin{abstract}
RESUMO: Este artigo tem o objetivo de apresentar o modelo comportamental de racionalidade limitada proposto por Herbert Simon para analisar o processo de tomada de decisão dos agentes em sistemas complexos, marcados pela não ergodicidade e pelo desenvolvimento de trajetórias em aberto. O artigo apresenta a noção de escolha logicamente consistente em contraponto à análise axiomática proposta pela teoria neoclássica. Propõe o uso de modelos de simulação em computador do tipo agent-based modeling (ABM) como um instrumental adequado à análise de sistemas complexos.
\end{abstract}

PALAVRAS-CHAVE: racionalidade limitada; sistemas complexos; modelos de simulação.

ABSTRACT: This article aims to present the behavioral model of bounded rationality proposed by Herbert Simon as a framework to analyze the process of decision-making of agents in complex systems, characterized by non-ergodicity and developing open trajectories. The article presents the notion of logically consistent choice and contrasts it with the axiomatic analysis of the neoclassical theory. It argues for the use of computer simulation models, like agent-based modeling (ABM) as a suitable tool for analyzing complex systems. KEYWORDS: bounded rationality;complex systems; simulation models.

JEL Classification: B41; B52.

\section{INTRODUÇÃO}

Este artigo tem o objetivo de apresentar o argumento de racionalidade limitada proposto por Herbert Simon como alternativa à visão neoclássica de racionali-

\footnotetext{
* Professores do Departamento de Economia, Universidade Estadual Paulista, Faculdade de Ciência e Letras -UNESP/FCL, Araraquara. E-mails: tmassaroli@fclar.unesp.br; e jrfucidji@fclar.unesp.br. Submetido: 5/Maio/2014; Aprovado: 4/Setembro/2015.
} 
dade, fundamentada na imposição de axiomas para a compreensão das motivações dos agentes econômicos. Na busca de elementos que permitam a interpretação da tomada de decisão dos agentes, o argumento de racionalidade substantiva ou maximizadora, desenvolvido no âmbito da corrente neoclássica, admite que os resultados das ações humanas possam ser inteiramente antecipados. A incorporação da incerteza no processo de raciocínio que assume racionalidade substantiva existe apenas como incerteza sobre a probabilidade de ocorrência dos eventos futuros (incerteza estrutural). A incerteza gerada como consequência da interação entre agentes, por outro lado, torna a abordagem de Simon bastante adequada para a análise de sistemas complexos.

$\mathrm{O}$ argumento de racionalidade limitada, proposto por Simon, foi construído a partir da premissa de dinâmica do sistema econômico, em que não apenas as ações dos agentes econômicos mudam ao longo do tempo, mas também o próprio ambiente em que atuam. Dessa forma, a capacidade de predição dos eventos futuros torna-se impossível, em virtude da incerteza sobre as condições futuras do ambiente. Além da incerteza quanto à probabilidade de ocorrência de eventos futuros, o argumento de racionalidade limitada assume também que os agentes não possuem capacidade de obter e processar todas as informações relevantes para a tomada de decisão, tendo em vista a complexidade do sistema em que estão inseridos. Portanto, racionalidade limitada não está apenas relacionada à incerteza estrutural, pois mesmo considerando uma situação hipotética de perfeita informação, a limitação dos agentes para processar este conjunto de informações inviabiliza a busca de resultados maximizadores. $\mathrm{O}$ artigo propõe que os modelos de simulação são ferramentas adequadas para a análise de sistemas complexos caracterizados por racionalidade limitada.

Além desta introdução, o artigo está estruturado em seis seções. A segunda seção apresenta a noção de escolha logicamente consistente utilizada na construção do modelo comportamental de racionalidade limitada, contraposta à análise axiomática proposta pela teoria neoclássica. A terceira seção discute a relação entre racionalidade limitada e busca de resultados satisfatórios (satisfacing) em oposição à busca, característica dos modelos tradicionais, de resultados ótimos globais. A quarta seção é dedicada à análise do processo de seleção de inovações ou novidades pelo ambiente, considerando-as como resultados não intencionais da interação entre os agentes, complicando o ambiente que norteia a tomada de decisão. A quinta seção apresenta a noção de racionalidade processual como uma sequência de procedimentos padronizados (ou algoritmos) que permite a estabilidade e a evolução em sistemas complexos. A sexta seção mostra as vantagens associadas ao uso de modelos de simulação baseados em agentes (ABM), comparativamente aos modelos analíticos tradicionais, para analisar sistemas complexos sujeitos à racionalidade limitada. A sétima seção apresenta algumas considerações finais. 


\section{A NOÇÃO DE ESCOLHA LOGICAMENTE CONSISTENTE E O ARGUMENTO DE RACIONALIDADE LIMITADA}

O modelo comportamental de racionalidade limitada, desenvolvido por Herbert Simon (1955), procura elaborar uma alternativa ao argumento axiomático tradicional de racionalidade, puramente abstrato, amparado na imposição de pressupostos e regras de inferência para extrair proposições normativas. $\mathrm{O}$ autor aponta as fragilidades do uso da lógica para encontrar algum padrão de comportamento dos agentes, coerente e infalível, para a resolução de problemas complexos.

A principal noção que fundamenta o modelo de racionalidade ilimitada ou substantiva é a de que não há conclusões sem premissas, o que torna o uso da lógica indispensável para a construção de uma teoria normativa, na medida em que o estabelecimento de regras de inferência permite obter leis gerais imutáveis com base em axiomas ou pressupostos induzidos de observações empíricas. No entanto, para Simon (1983, p. 15), embora a teoria da racionalidade ilimitada ampare-se no uso da lógica para extrair conclusões normativas, as regras de inferência utilizadas são estabelecidas arbitrariamente e, nesse sentido, os processos que transformam os axiomas em resultados são impostos sem qualquer fundamentação lógica.

A racionalidade substantiva implica que todas as contingências futuras podem ser antecipadas. ${ }^{1}$ Esta implicação com relação à ocorrência de eventos futuros somente pode ser justificada se forem observadas duas condições. Em primeiro lugar, assume-se que o sistema econômico é ergódico, o que inviabiliza a existência de surpresas genuínas no futuro. Em segundo lugar, assume-se uma homologia entre o mundo do modelo e o mundo real. Porém, dada a sua natureza axiomática, o suposto de racionalidade substantiva presta-se apenas à prova lógica, mas não empírica. A alternativa proposta por Simon - o argumento de racionalidade limita$\mathrm{da}$ - foi elaborada a partir da refutação destes dois princípios que fundamentam o modelo tradicional de racionalidade.

Mesmo assumindo que os axiomas não são arbitrários, os resultados obtidos a partir do processo de inferência não podem ser infalíveis. Como o sistema econômico muda constantemente, a imprevisibilidade de eventos futuros faz com que surpresas genuínas sejam frequentes. Portanto, não existe apenas incerteza a respeito da probabilidade da ocorrência de um determinado evento - incerteza estrutural, sobre a forma da distribuição de probabilidades de eventos -, mas também incerteza genuína quanto às mudanças no ambiente de decisão e o hiato que provocam entre a dificuldade da tomada de decisão e a competência do agente (Heiner, 1983; Vercelli, 1991, p. 79).

Simon (1983, pp. 33-34) descreve alguns mecanismos, presentes nos seres humanos, que tornam plausível o modelo comportamental de racionalidade limitada.

\footnotetext{
${ }^{1} \mathrm{~A}$ incerteza pode incidir apenas sobre a probabilidade da ocorrência de eventos futuros e, portanto, a racionalidade substantiva admite a presença de incerteza estrutural, mas não de incerteza genuína no sentido de Heiner (ver abaixo).
} 
O primeiro desses mecanismos é a capacidade de concentração dos indivíduos nos problemas que necessitam de atenção imediata. O segundo mecanismo é a capacidade humana de produzir alternativas de ação, no sentido de procurar alternativas viáveis ou o aprimoramento daquelas que já existem. O terceiro mecanismo, e de maior relevância para o ponto discutido nesta seção, é a capacidade humana de adquirir fatos e inferir a partir desses fatos; neste aspecto, o autor substitui o uso de axiomas pela noção de escolha logicamente consistente. Uma escolha será racional se for consistente com as informações que os agentes econômicos têm disponíveis no momento da tomada de decisão. Isso não significa que as decisões sejam coerentes, pois dependem da ordem em que são apresentadas as alternativas. Por exemplo, um indivíduo pode escolher a alternativa $\mathrm{A}$ em vez de $\mathrm{B}$, quando $\mathrm{A}$ for apresentada antes de $B$. Caso a alternativa $B$ seja apresentada em primeiro lugar, o mesmo indivíduo pode mudar sua ordem de preferência, escolhendo B em vez de A.

As informações utilizadas pelo agente para a tomada de decisão são baseadas em fatos muito subjetivos, como as percepções que os indivíduos têm acerca do ambiente em que vivem. A racionalidade dos agentes depende de suas crenças, e estas crenças dependem das informações disponíveis no momento de suas ações. Neste sentido, um conjunto de informações completamente deturpadas pode gerar comportamento racional, desde que as ações de um grupo de indivíduos sejam amparadas por estas informações.

Para Tisdell (1996, pp. 23-24), embora a consistência lógica garanta a racionalidade das ações dos indivíduos, ela é insuficiente para conduzi-los a situações ótimas, no sentido paretiano, ou seja, a consistência lógica não garante o bem-estar do agente econômico. As crenças individuais podem ser logicamente consistentes e, portanto, racionais, mas os indivíduos podem estar tão mal informados que são incapazes de agir em seu próprio benefício. Portanto, é possível qualificar a escolha racional como um ótimo apenas relativo às crenças dos indivíduos e às informações disponíveis no momento da tomada de decisão. Para entender as escolhas individuais é necessário conhecer os objetivos e percepções dos agentes.

O modelo comportamental de racionalidade limitada procura resgatar elementos da psicologia para compreender como de fato as pessoas tomam decisões. Ain$\mathrm{da}$ que os pressupostos psicológicos tenham fundamentado a teoria utilitarista do século XIX, eles foram substituídos pela tentativa de desenvolvimento de um conceito de racionalidade pura, que conseguisse acomodar todas as situações que envolvessem escolhas. Conforme apresentado em Sugden (1991, p. 757), a noção de probabilidade subjetiva definida em termos de preferências sobre apostas, desenvolvida por Ramsey nos anos 1930, culminou com a criação da teoria da utilidade esperada de Von Neumann e Morgenstern, dando origem à moderna teoria da escolha racional. A lógica tem nesta teoria a função apenas de servir como um critério para identificar inconsistência entre crenças, bem como derivar crenças complicadas a partir de outras mais simples. Nesta teoria a ação, objeto da escolha do indivíduo, está intimamente relacionada com um conjunto de consequências, e sempre haverá uma única consequência para cada "estado do mundo" ou conjunto de pressupostos sobre o ambiente. Como as preferências do indivíduo são rela- 
tivas às suas ações, a teoria da escolha racional utiliza a lógica para definir um conjunto de axiomas capazes de estabelecer condições de consistência sobre preferências. Se as preferências de um indivíduo são satisfeitas por esses axiomas, então tais preferências podem ser representadas por uma única função de utilidade, que permite a obtenção de um nível de utilidade para todas as consequências, e por uma única função de probabilidade, que permite obter a probabilidade conjunta associada a cada evento ou estado do mundo. Estas funções são utilizadas para determinar um índice de utilidade esperada para cada ação. Sendo assim, dado um conjunto de ações possíveis, aquela com maior utilidade esperada será a preferida.

Para Sudgen (1991), o ponto central desta teoria é que escolhas revelam preferências e, embora a análise das preferências tenha sido o ponto de partida, o resultado alcançado não foi uma teoria da preferência racional e sim a teoria da escolha racional. A razão é um instrumento útil apenas quando a estrutura do problema é determinada, não existindo a possibilidade de apresentar o mesmo problema com uma estrutura diferente. Portanto, a teoria da escolha racional não permite afirmar que uma ação pode ser racionalmente escolhida em qualquer problema de decisão, mas apenas afirmar que a escolha de determinada ação, no contexto de determinado problema, é consistente com a escolha de outra ação em outro contexto. A proposta da teoria é identificar a consistência de determinada escolha com uma outra para então obter um padrão de consistência das escolhas e, a partir daí, derivar medidas de utilidade e probabilidade para as mesmas. Um ideal de racionalidade seria aquele em que todas as ações estivessem amparadas na razão, o que significaria autonomia do indivíduo no sentido de que ele é capaz de agir de acordo com os princípios que escolheu para si próprio (Sugden, 1991, p. 760).

Para Gigerenzer (2008, p. 20), o foco da lógica é sobre a verdade, pois todos os sistemas que investigam a cognição e são inspirados na lógica avaliam a capacidade do indivíduo de resolver silogismos, mantendo a consistência entre crenças. No entanto, o uso da lógica nas análises descritivas e normativas encontra sérias deficiências, pois muitas das consequências deduzidas das premissas desconsideram os processos psicológicos dos agentes. Portanto, o comportamento sistemático que se espera de um indivíduo que utiliza a lógica para extrair conclusões com base em premissas diverge significativamente do comportamento observado. Nesse sentido, os modelos que utilizam heurísticas cognitivas, ou atalhos cognitivos para reduzir a complexidade dos problemas a serem solucionados, ao contrário de instrumentos puramente lógicos, mostram que, na grande maioria dos casos, os indivíduos tomam decisões acerca de problemas mal definidos, com múltiplos objetivos e sem conhecimento sobre probabilidades ou função de utilidade, impedindo, portanto, que o uso da lógica seja capaz de encontrar uma solução ótima.

Colinsk (1996) apresenta uma série de exemplos em que a tomada de decisão dos agentes segue uma norma de conduta totalmente diferente do padrão de racionalidade maximizadora proposto pela teoria econômica ortodoxa. Em muitos experimentos sobre o comportamento individual, contatou-se que os agentes frequentemente possuem preferências intransitivas, falham em perceber os efeitos da lei dos grandes números, falham em reconhecer dominância estatística, erram ao 
atualizar probabilidades com base em nova informação ou mesmo ignoram informação relevante. Há uma extensa lista de situações em que os agentes não possuem comportamento racional maximizador e, portanto, as regularidades que são observadas, relativas ao padrão de tomada de decisão, não podem ser compreendidas com base no postulado de racionalidade forte, mas sim a partir de alguma heurística adotada pelos agentes com o objetivo de contornar limitações cognitivas e economizar custos de deliberação. Neste sentido, Heiner (1983) argumenta que a economia ortodoxa está ironicamente sujeita a um problema de má especificação, pois as regularidades que a teoria tenta explicar com base na otimização podem desaparecer caso os agentes consigam, de fato, maximizar.

Em Colinsk (1996, p. 681) racionalidade deve ser de alguma forma modelada como adaptação dinâmica, pois ao utilizar intuição e observação os agentes passam a apresentar padrões de comportamento adaptativos no momento em que interagem com determinado ambiente. As heurísticas cognitivas são armazenadas na memória e, portanto, a mente funciona como uma ferramenta adaptativa, com várias heurísticas adaptadas a classes específicas de problemas. Neste ponto, o autor destaca a importância do uso de modelos computacionais para a análise do processo de tomada de decisões em sistemas complexos, já que o computador tem a capacidade de armazenar heurísticas e identificar a classe de problema em que uma determinada heurística é aplicada (ver a sexta seção). Modelos baseados em heurísticas são capazes de explorar capacidades que evoluem naturalmente no homem, como a capacidade da memória de armazenar e reconhecer padrões de solução de problemas utilizados no passado, o que não seria possível com o cálculo estatístico.

\section{RACIONALIDADE LIMITADA E SOLUÇÕES SATISFATÓRIAS}

A compreensão sobre como a razão influencia as escolhas é o elemento central da teoria econômica desde o pensamento iluminista de Hume no século XVIII, de que a razão deve atender aos desígnios do sentimento puro. Uma vez que toda ação é motivada por algum tipo de paixão, a razão sozinha não é capaz de impulsionar alguma ação (Sudgen, 1991, p. 753). A ideia de que a ação é motivada pelo desejo, sendo o estado de desejo algo inerente ao homem e não resultado de sua reflexão racional, é utilizada para a construção da teoria utilitarista do século XIX.

Neste ponto, uma distinção importante deve ser feita entre razão e racionalidade. De um lado, a razão é a capacidade de julgamento da mente humana, formadora de suas crenças e deliberações. De outro, a racionalidade é um instrumento, uma espécie de dispositivo para ajustar meios disponíveis a fins dados (racionalidade instrumental). A teoria utilitarista, supõe dados os desejos e a racionalidade substantiva trata de indicar os meios para o atingimento dos estados almejados (o resultado do cálculo maximizador). Ora, neste sentido, a racionalidade substantiva é internamente consistente e, contudo, permanece uma concepção simplista da razão humana. Esta questão será explorada por Simon. Um dos aspectos principais da teoria da racionalidade 
limitada é questionar o uso da racionalidade em modelos analíticos para a obtenção de soluções maximizadoras. Uma dessas limitações é o reconhecimento de que há vários elementos do acaso que mudam o resultado das ações, sendo a razão instrumental insuficiente para identificar previamente todas as contingências futuras de determinada ação. Se na tentativa de alcançar um determinado objetivo o indivíduo utiliza a razão, apenas conseguirá encontrar formas de chegar até este objetivo, mas nunca a certeza de que conseguirá alcançá-lo. Há, portanto, uma utilidade limitada do uso do raciocínio. Em Simon (1983, pp. 7-8), “[a razão] é uma arma mercenária que pode ser posta a serviço de qualquer dos nossos objetivos". A alternativa, o modelo de utilidade esperada subjetiva, tem "dificuldades que tornam impossível utilizá-la na tomada de decisão por humanos reais. O modelo supõe que o tomador de decisões é capaz de contemplar, de modo completo, o futuro perante ele. Ele entende a série de escolhas alternativas que estão disponíveis, não apenas no momento, mas em todo o panorama do futuro. Ele entende as consequências de suas escolhas, a ponto de ser capaz de atribuir uma distribuição conjunta de probabilidades aos estados futuros do mundo" (Simon, 1983.p. 13).

$\mathrm{O}$ argumento de racionalidade limitada assume a existência de um "mundo único" composto por milhões de variáveis que, em princípio poderiam afetar-se mutuamente, mas que não o fazem na maior parte do tempo. Esta suposição tem implicações diretas sobre a análise da relação entre alternativas e consequências. Ao analisar esta relação, é preciso distinguir entre aquela que está na cabeça do tomador de decisão e aquela que existe "objetivamente", dado que o tomador de decisão possui apenas um conhecimento fragmentado das condições em torno de suas ações, não sendo capaz de estabelecer uma relação exata entre alternativas e consequências (Koblitz, 2008, p. 267; Simon, 1986, pp. S211-S212). Embora seja plausível que exista no mundo uma rede densa de inter-relações, a observação permite concluir que, na maioria dos casos, há apenas um número reduzido de variáveis que se conectam diretamente. A conexão entre as variáveis pode ser feita por meio de mecanismos específicos e, portanto, uma variável pode ser incluída num subsistema, desde que seja selecionado um mecanismo por meio do qual ela consiga exercer algum tipo de influência sobre aquele subsistema. A análise destes mecanismos torna-se mais relevante que a análise de suas prováveis consequências, pois permite compreender os processos que orientam a tomada de decisão, a partir do reconhecimento de que os problemas podem ser separados em seus componentes básicos. Em Simon (1983, p. 33), o homem somente foi capaz de se desenvolver num mundo extremamente complexo porque conseguiu elaborar procedimentos bem definidos para a tomada de decisão.

A característica crucial destes procedimentos refere-se à tomada de decisões na forma sequencial. No modelo comportamental de racionalidade limitada, as escolhas não precisam ser infinitamente longas, de forma a abranger a interdependência entre todas as variáveis de um "mundo único". Separar os problemas em seus componentes básicos significa estabelecer prioridades, ou seja, resolver, primeiramente, os problemas mais urgentes e postergar os demais. Nesse sentido, as escolhas que abrangem todos os valores humanos, em que cada problema está interligado 
com todos os problemas do mundo, são completamente desnecessárias. Para Simon (1983, p. 21), “O animal existente em nós nos leva a agir de forma sequencial em relação a cada uma de nossas necessidades, criando, instintivamente, uma escala de prioridades para nossas necessidades". A visão comportamental descreve, de fato, a maneira como os indivíduos tomam suas decisões e se desenvolvem em um sistema complexo. Em alguns casos, a escolha de determinado procedimento para a solução de problemas é interpretada como descoberta súbita, sem nenhum explicação lógica. É a simples experiência do "Eureka!". São situações em que as pessoas encontram, de repente, a solução para seus problemas. Esta experiência é explicada pela capacidade da memória de armazenar os conhecimentos adquiridos no passado e resgatá-los por meio da intuição. Em Simon (1983, p. 21), o pensamento intuitivo é explicado quando o indivíduo adquire experiência considerável em algum campo da ciência. Neste sentido, a descoberta súbita é a "cognição quente", que tende a evocar emoção ao resgatar aquilo que está armazenado na memória.

Desenvolver um procedimento ou uma forma de cálculo para traçar um bom curso de ação é compatível com o modelo de racionalidade limitada, porém este procedimento nunca garantirá o ótimo global. Segundo Tisdell (1996, p. 31; Simon, 1986, 1999), os agentes econômicos não procuram maximizar uma função-objetivo, mas sim obter resultados satisfatórios. A racionalidade que permeia as decisões humanas é a racionalidade processual. É possível encontrar um resultado aproximado que permita traçar um bom curso de ação. A noção de racionalidade substantiva, ao contrário, apoia-se na premissa de que os indivíduos conseguem escolher o curso de ação exato para alcançar objetivos maximizadores, ou seja, ótimos globais.

O modelo comportamental de Simon (1983, pp. 66-68; 1999, pp. 26-30) afirma que, mesmo diante de certeza absoluta com relação ao futuro, é impossível maximizar uma função-objetivo. As limitações para obtenção e processamento de informações, características dos seres humanos, tornam factíveis apenas a existência de ótimos locais. Neste contexto, a racionalidade processual, ao criar modelos aproximados do mundo real, auxilia na busca de uma solução satisfatória. Simon (1999, p. 29), utiliza exemplos dos métodos de pesquisa operacional (PO) e inteligência artificial (IA) para ilustrar como uma firma pode obter resultados satisfatórios.

A pesquisa operacional trabalha com procedimentos (algoritmos) para auxiliar a resolução de problemas envolvendo incerteza. No entanto, os procedimentos da PO somente podem ser aplicados quando limitações ao raciocínio são impostas. A programação linear é um bom exemplo. O mundo real deve ser representado pela imposição de restrições lineares, para que a programação linear seja aplicada. A despeito desta limitação, os resultados obtidos pela PO, de acordo com Simon (1999, p. 29), são soluções satisfatórias no mundo real, embora no mundo hipotético e reformulado da $\mathrm{PO}$ tais resultados sejam obtidos a partir do pressuposto da maximização. No caso dos métodos de IA, ao contrário da PO, o objetivo é encontrar soluções satisfatórias e não ótimas. Embora a inteligência artificial também seja uma aproximação do mundo real, sua aplicação é feita com a incorporação de detalhes do mundo real e não pela imposição de restrições. Dessa forma, a IA per- 
mite que sejam formulados problemas mais complexos, embora não tão bem estruturados quanto aqueles formulados com base no método da PO.

A incorporação de racionalidade processual ao modelo comportamental proposto por Simon $(1999$, p. 29) é justificada pela necessidade dos agentes econômicos de encontrar soluções suficientemente boas para seus problemas, uma vez que, diante da complexidade do mundo real, as soluções ótimas raramente estão disponíveis.

\section{O PROCESSO DE SELEÇÃO}

$\mathrm{Na}$ tentativa de encontrar soluções suficientemente boas para os problemas que enfrenta, o agente procura estabelecer determinadas regras heurísticas, com o intuito de amenizar os efeitos da incerteza. Para Gigerenzer (2008) este processo de seleção de heurísticas deve ser analisado com base na ferramenta adaptativa, isto é na capacidade da mente de adaptar o comportamento presente ao comportamento passado, resgatando heurísticas utilizadas no passado para resolver problemas atuais ou mesmo criando novas regras de comportamento a partir de padrões de solução de problemas já conhecidos e utilizados. Segundo o autor, a seleção é guiada por aprendizado, obtido por esforço individual (esforço de tentativa e erro), convívio social e habilidades que evoluem naturalmente nos indivíduos (aprendizado evolucionário). A análise dos processos de busca e seleção de rotinas é particularmente importante para a teoria econômica evolucionária que, assentada sobre o princípio da racionalidade limitada, é capaz de reconhecer que os limites impostos à taxa de mudança técnica estão associados à habilidade limitada dos agentes para perceber e explorar oportunidades tecnológicas que permitam a seleção de rotinas novas ou aprimoradas para melhoria dos processos de produção.

Um ponto a ser considerado é a pertinência da analogia dos processos de busca e seleção na economia com os mecanismos da biologia darwinista. Tal analogia deve ser apenas parcial, considerando que a variação no ambiente econômico não é totalmente cega, elementos como intencionalidade e insights têm um papel muito mais importante na evolução econômica que na evolução biológica, não sendo possível a analogia entre variação econômica e mutação biológica.

$\mathrm{Na}$ teoria darwinista, variação e seleção são mecanismos independentes, a variação ocorre de forma cega, baseada no surgimento de mutações, e posteriormente as mutações bem-sucedidas são selecionadas para se reproduzirem no ambiente. Porém, ao serem aplicados à análise da evolução econômica, observa-se total dependência entre os mecanismos de variação e seleção. Como os indivíduos são capazes de antecipar resultados adversos e tomar medidas de precaução, a seleção deixa de operar inteiramente ex-post, já que o indivíduo é capaz de seletivamente excluir alguns dos resultados que não são desejados.

Em Vromen (2004), a atividade de busca é parcialmente cega, no sentido de que embora a busca seja guiada por rotinas, o sucesso desta atividade nunca é garantido e somente os resultados bem-sucedidos são escolhidos. Como a infor- 
mação disponível para o agente é limitada ou distorcida e seu processamento envolve custos consideráveis, as ações dos agentes, embora motivadas, normalmente são incapazes de produzir resultados maximizadores. A questão da pertinência dos resultados maximizadores é amplamente discutida em Vromen (1995, cap. 2 e 4). $\mathrm{O}$ autor argumenta que a incompatibilidade entre a teoria neoclássica da firma e a crítica antimarginalista não é clara, já que o comportamento rotineiro da firma pode ser explicado por um processo evolucionário no qual as regras menos lucrativas são substituídas por outras mais lucrativas. "This is a possibility Hall and Hitch also allude to in their interpretation of their empirical findings: it is possible (in principle, at least) that maximum profits are an evolutionary 'by-product' of the application of the 'full cost' rule" (Vromen, 1995, p. 17). Neste processo evolucionário, as melhores regras são selecionadas enquanto outras são eliminadas ou passam a ter importância reduzida junto à população e, nessa lógica, a maximização do lucro seria apenas um resultado do comportamento rotineiro da firma e, portanto, comportamento rotineiro não inviabiliza a análise marginalista. $\mathrm{O}$ ponto que difere a abordagem evolucionista da firma da abordagem marginalista é que na busca de determinado alvo, as ações de agentes mais bem preparados podem não ser selecionadas, simplesmente por não encontrarem oportunidades de desenvolvimento ao longo do processo de busca, portanto nem sempre ao seguir rotinas os agentes encontrarão os melhores resultados, como observado nas situações em que a economia fica aprisionada (loked-in) a uma tecnologia inferior. Ademais, seguindo a abordagem de Alchian (1950), a análise marginalista é aplicável apenas quando se é capaz de definir ex ante uma função de preferência subjetiva com relação a risco que garanta o melhor resultado possível. O problema é que na presença de incerteza, informação incompleta e conhecimento imperfeito, o resultado maximizador torna-se improvável.

A partir desta ideia, propomos utilizar o arcabouço evolucionista, que explica como o comportamento adaptativo dos agentes muda ao longo do tempo por um processo de seleção. A mudança é determinada por alterações nas características que possuem significância seletiva para a população relevante. Neste sentido, o processo gerador de mudanças é responsável pela criação de diversidade e desordem no sistema. A dinâmica que caracteriza sistemas complexos deriva justamente do surgimento desta diversidade, que deve ser necessariamente entendida como surgimento de novidades ou inovações. ${ }^{2}$ Variedade e seleção fazem parte do mesmo processo evolutivo e, portanto, possuem características complementares, pois a desordem gerada pela introdução de variedade é necessária para que o processo

\footnotetext{
${ }^{2}$ Vale destacar que, para os pós-leynesianos, o conceito de incerteza fundamental ou forte dá ênfase à introdução de inovações (Dequech, 1997, p. 32). Quando Simon vê uma aproximação entre racionalidade limitada e incerteza, esta decorre da incapacidade de previsão de consequências e, embora ele admita a introdução de mudança nos algoritmos de decisão (rotinas) das firmas, não especifica nenhum mecanismo gerador de mudança (ver a quinta seção). Por isso, os pós-keynesianos rejeitam a aproximação entre os dois conceitos (ver Dequech, 2001, e Dunn, 2001). Este problema é apontado por Koblitz (2008, pp. 263-264).
} 
seletivo ocorra, escolhendo as características que melhor se ajustam ao ambiente e restabelecendo, assim, a estabilidade do sistema (Fonseca, 2001, pp. 3-4).

Metcalfe et al. (2002, pp. 97-98) ressaltam que evolução e adaptação não podem ser reduzidas à análise apenas dos mecanismos de variedade e seleção. Uma das distinções entre a teoria evolucionista e a teoria neoclássica reside no tratamento da geração de variedades. Para a corrente neoclássica, a geração de variedade é extrínseca ao sistema e, portanto, não pode ser explicada como um fenômeno endógeno. Nesta visão, a variedade, ou inovação, é interpretada como um choque externo que causa desvios temporários do sistema em relação a uma situação estável, entendida como equilíbrio de longo prazo. O sistema econômico possui atratores que o conduzem sempre para uma situação de equilíbrio de longo prazo independentemente dos solavancos sofridos ao longo do caminho, os quais nunca são interpretados como fatores causados pelo próprio sistema. Para a teoria evolucionista, ao contrário, as variedades emergem do próprio sistema, sendo explicadas pela interação dos agentes que o compõem. O processo de interação entre ofertantes e demandantes no mercado, por exemplo, faz com que os agentes aprendam o que produzir e de quem comprar.

O aprendizado torna-se um elemento importante para a compreensão do sistema econômico quando a análise estática é substituída pela análise dinâmica, com base no reconhecimento de que ao longo do tempo os agentes experimentam novos eventos e adquirem novas informações que influenciarão seu comportamento e suas expectativas sobre a atividade econômica no futuro. Deve-se notar que, a menos que um agente inovador (um agente schumpeteriano) ou um mecanismo de aprendizado seja introduzido na análise, o surgimento de inovações ou "novidades" deverá ser creditado a algum fator aleatório não especificado. $\mathrm{Na}$ interpretação evolucionista de Simon, aqui proposta, a geração de variedade não pode ser inteiramente aleatória porque é também em parte determinada pelo aprendizado em experiências prévias.

O processo de seleção de variedades também é fortemente influenciado pelo acúmulo de experiências. Segundo Azevedo (1996), embora o método de tentativa e erro $^{3}$ seja crucial ao processo de seleção para Simon, a capacidade da memória para registrar as consequências de ações realizadas no passado permite que a experiência prévia exerça uma função complementar ao mecanismo de seleção por tentativa e erro. Dada a semelhança entre as situações no passado e no presente, a experiência prévia é útil para economizar esforços. A combinação destes dois elementos é um fator explicativo da estabilidade de sistemas complexos, mesmo diante dos solavancos provenientes de eventos imprevistos, característicos de tais sistemas.

Em Simon (1999, p. 44), um segundo fator que explica a estabilidade desses sistemas é o predomínio de hierarquias, em contraponto aos mercados, em am-

\footnotetext{
${ }^{3}$ O método de tentativa e erro de Simon assemelha-se ao proposto por Popper (1978, p. 16), que consiste em experimentar possíveis soluções para os problemas, e cada solução proposta deve ser passível de crítica e tentativa de refutação para atender ao critério de cientificidade.
} 
bientes marcados por grande incerteza. Coordenação e padronização, características do ambiente organizacional, garantem mais eficiência que os mercados. A eficiência do ambiente organizacional é fruto da identificação criada dentro das organizações. Por causa da racionalidade limitada e da complexidade dos sistemas, os indivíduos têm dificuldade para compreender o mundo, sendo necessário criar uma "imagem simplificada do mundo, isto é, uma interpretação do ambiente a partir do ponto de vista, dos interesses e objetivos da organização" (idem). Os membros de uma organização com frequência se identificam com, e são levados a perseguir, objetivos organizacionais à custa de seus próprios objetivos. Nesse contexto, indivíduos que aceitam as regras e seguem rotinas organizacionais com facilidade têm grande vantagem em relação àqueles que não o fazem. Rotinas e procedimentos, além de garantirem a estabilidade das hierarquias, permitem sua evolução em um sistema econômico altamente complexo. A necessidade de rotinas para o desenvolvimento do processo evolutivo aponta para o que se pode chamar de miopia da evolução. Ainda que um sistema econômico se desenvolva, não é possível garantir que ele atingirá objetivos previamente definidos ao final do processo. A ideia de miopia do processo evolutivo é particularmente relevante para analisar as atividades tecnológicas das firmas, dada a imprevisibilidade dos resultados esperados a partir dos esforços de pesquisa e desenvolvimento.

Em sistemas complexos, caracterizados pela não ergodicidade, ${ }^{4}$ as firmas constroem modelos internos baseados em suas experiências tecnológicas passadas para estimar a lucratividade de seus projetos de inovação e, dessa forma, permitem alguma coevolução entre a população de novos projetos tecnológicos e os modelos necessários para avaliar seu potencial de lucratividade. Isso decorre do fato de que um novo projeto tecnológico somente será adotado se ele for mais lucrativo que a tecnologia corrente da firma. Assim, ao construir modelos internos para a avaliação dos diferentes projetos, a firma aumenta sua probabilidade de obter sucesso a partir do esforço inovativo. "[...] O uso de modelos internos, desenvolvidos para que seja possível eleger o projeto a ser implementado, aumenta substancialmente o desempenho da firma, comparativamente ao caso em que qualquer projeto desenvolvido é implementado" (Dawid, 2005, p. 20). Dada a miopia evolutiva, a construção de regras e procedimentos internos, baseados nas experiências tecnológicas passadas, atenua os efeitos da incerteza sobre o resultado do esforço inovativo, aumentando a probabilidade de obter inovações bem-sucedidas.

Simon $(1999$, p. 48) utiliza esta noção de miopia do processo evolutivo para contestar a noção neoclássica de máximo global. A constante mudança dos sistemas e, consequentemente, a incerteza com relação aos seus estados futuros, faz com que

\footnotetext{
${ }^{4}$ A ergodicidade corresponde à homogeneidade temporal das diferentes classes de eventos e das condições nas quais eles ocorrem, implicando, no que diz respeito às probabilidades estatísticas, que a frequência do evento no futuro seja a mesma do passado. Em condições de não ergodicidade e incerteza com relação ao futuro, a homogeneidade dos eventos não será mantida; logo, a frequência do evento no futuro não coincidirá com sua frequência no passado. Dessa forma, a divergência entre valores $e x$ ante e ex post denota a existência de mudanças no sistema (Herscovici, 2004, p. 820).
} 
o abandono de uma situação estável em direção a uma nova situação seja sempre míope. A ação dos agentes econômicos, embora motivada pela busca de maiores lucros, não garante que a situação encontrada ao final do processo seja estável. Ao sair de um ponto em direção a outro, o agente econômico pode não ter noção do abismo que existe entre eles. Existem pontos de máximo em sistemas complexos, porém são apenas máximos locais. Para Simon (1999, p. 47), máximos locais são sistemas em que cada subsistema está otimamente adaptado a outro subsistema ao redor, de forma que o equilíbrio é apenas local.

$\mathrm{Na}$ teoria comportamental de Simon (de modo compatível com a teoria evolucionista), o uso de procedimentos padronizados pelas firmas tem o objetivo de absorver a incerteza. Os procedimentos utilizados pelas firmas podem ser interpretados como algoritmos que permitem a tomada de decisões diárias. Estas decisões tornam-se rotineiras na medida em que geram resultados bem-sucedidos e, afinal, são repassadas para gerações sucessivas de executivos e empregados. Seguindo esta lógica, evolução é tudo aquilo que promove mudanças nesses algoritmos. Uma nova ideia pode ser incorporada ao algoritmo desde que possua condições de gerar resultados bem-sucedidos 5 , embora as consequências da incorporação dessa nova ideia não estejam determinadas a priori.

\section{EVOLUÇÃO COMO UM PROCESSO ALGORÍTMICO}

O modelo comportamental de racionalidade limitada, exposto até aqui, fornece explicações sobre como os indivíduos tomam decisões, mais precisamente, quais são os processos utilizados pelos agentes econômicos para encontrar alternativas de ação. Tais procedimentos podem ser entendidos como algoritmos, os quais determinam a evolução do sistema. O conceito de algoritmo aplicado à evolução foi utilizado pela primeira vez na teoria de Darwin para explicar o mecanismo de seleção natural em sistemas biológicos.

$\mathrm{Na}$ teoria darwinista, a evolução ocorre por meio de um processo formal que tem, necessariamente, um resultado específico, embora não seja determinado $a$ priori. Em um sistema biológico, as variedades geradas por meio de mutações aleatórias são selecionadas pelo ambiente. O processo pode ser visto como a aplicação de um algoritmo seletivo utilizando o acaso ou a aleatoriedade (Dennett, 1998, p. 55).

O algoritmo é um processo formal em que há consistência lógica entre as informações recebidas e os resultados gerados. O desenho de um objeto complexo, por exemplo, uma face humana, necessita que as informações sejam fornecidas em sequência, de forma hierárquica. Primeiro desenhar o contorno da face e depois

\footnotetext{
${ }^{5}$ Ver Simon (1999, p. 48) ao mencionar a teoria evolucionista de Nelson e Winter (1982). Note-se que esta é uma concepção específica e bastante ampla de inovação. Ver as objeções pós-keynesianas (nota 2).
} 
inserir as características, como os olhos, a boca, as orelhas, o nariz, por fim, os detalhes de cada característica, como a cor dos olhos, o formato do nariz etc. Portanto, algoritmos são decisões tomadas de forma sequencial e hierárquica, gerando procedimentos padronizados ou rotinizados. Em Simon (1999, p. 206), um sistema complexo é constituído de diversos subsistemas interligados, em que existem milhões de variáveis e cada uma está relacionada com todas as demais. Para garantir estabilidade em um sistema desse tipo, é necessário que as decisões sejam tomadas de forma sequencial com o objetivo de obter resultados satisfatórios.

As informações passadas devem sempre orientar as decisões futuras para que haja evolução. Na memória, a mudança é adaptativa, pois ela atualiza o conhecimento sobre o mundo real e adiciona novos conhecimentos. Analogamente, a memória consegue adicionar novos procedimentos que visam ao aperfeiçoamento daqueles existentes.

Além de armazenar informações do passado, a memória também tem a propriedade de adicionar novos procedimentos, diante de mudanças no ambiente, aos algoritmos previamente definidos. Sendo assim, a incorporação das mudanças ambientais aos procedimentos adotados no passado pode fornecer resultados diferentes daqueles obtidos anteriormente (Simon, 1999, p. 106). Ainda que o processo de busca seja orientado por algoritmos bem-definidos, ele não necessariamente estará associado à solução de problemas complexos que possuem um objetivo definido. Ou seja, as respostas para um determinado problema podem ser determinadas a partir de modificações de um algoritmo bem sucedido no passado, embora não seja possível definir quais respostas serão encontradas no futuro. O processo de descoberta consiste justamente na busca de soluções que não podem ser definidas a priori.

Podemos associar essas ideias a alguns métodos de pesquisa. Métodos como a inteligência artificial e os programas de simulação são organizados como hierarquias de rotinas e sub-rotinas. Modificações nesses modelos aproximados do mundo real implicam alterações nessas rotinas e sub-rotinas. Simon (1999, p. 102) utiliza a lógica dos sistemas de produção, um modelo anterior à inteligência artificial e aos programas de simulação, para explicar como alterações nos algoritmos promovem mudanças. Um sistema de produção consiste de diversas produções dadas. Cada produção é definida como um conjunto de condições e um conjunto de ações. As ações contidas em uma produção somente serão executadas quando certas condições forem satisfeitas, de forma que cada produção opera independentemente das demais. Também é possível utilizar a noção de sistema de produção para entender o funcionamento de um sistema que estimula a cognição humana. Neste caso, o sistema deveria ser constituído de dois tipos de produção. No primeiro tipo a condição é fornecida por elementos que estão contidos na memória e, no segundo tipo, os elementos que definem a condição são as percepções do indivíduo sobre o ambiente externo. No primeiro caso a ação é condicionada por um objetivo predeterminado, enquanto no segundo caso a ação dependerá da percepção do indivíduo acerca do ambiente.

A representação de um sistema cujo comportamento é determinado por pro- 
duções baseadas em percepções exemplifica como um algoritmo pode ser utilizado para a solução de problemas que não possuem objetivos predefinidos. Embora a decisão do agente seja motivada, a ausência de conhecimento codificado acerca do problema a ser resolvido faz com que suas ações sejam míopes, o que o impede de formalizar um objetivo a ser perseguido. Como Simon explica: "Para descobrir ouro não é necessário nem mesmo olhar para ele (embora com frequência o seja) e se a prata ou o cobre forem descobertos ao invés de ouro, este resultado também será positivo. O teste de que alguma coisa nova foi descoberta é realizado quando alguma coisa emerge que não poderia ser prevista com certeza e esta coisa nova tem algum tipo de valor ou interesse".

A descoberta só ocorre quando o agente consegue encontrar uma sequência lógica de procedimentos que permita chegar a um determinado resultado. $\mathrm{O}$ agente econômico, ao seguir o procedimento estabelecido por regras, não conhece os resultados de sua ação. Portanto, o algoritmo permite que se tenha um método de pesquisa, mas este não determina, previamente, quais os resultados alcançados por segui-lo (Simon, 1999, p. 106; Rizzello, 1999, p. 49). A aplicação de simulação em computador à análise de sistemas complexos segue exatamente este princípio, ou seja, o simulador estabelece as regras de comportamento e as condições iniciais, mas não exerce qualquer influência sobre o resultado final obtido a partir das várias rodadas de simulação. Este ponto será tratado na próxima seção.

A decisão sobre qual procedimento seguir não é apenas baseada nas experiências de sucesso de um agente individual, mas sim no conjunto de agentes que optaram por um determinado procedimento no passado e obtiveram resultados bem-sucedidos. Em um sistema complexo, ainda que as decisões não sejam tomadas coletivamente, cada agente escolhe suas ações a partir da observação do mundo ao seu redor. Em Acha e Cusmano (2004, p. 14), um sistema complexo pode ser descrito como adaptativo no sentido de que sua evolução é guiada por mecanismos de aprendizado e, portanto, o sistema coevolui com os agentes nele inseridos. Por meio de interações entre os agentes, norma e ordem podem emergir no interior do sistema, permitindo sua estabilidade. Sistemas complexos adaptativos tentam representar a complexidade do mundo fora do modelo, ao extrair possíveis regularidades do ambiente representado. Um sistema adaptativo pode ser representado por uma hierarquia, como a firma, em que a emergência de regras permite sua evolução. Por outro lado, ao considerar a economia como um todo, regras de interação social ou instituições emergem da complexidade como mecanismos necessários para garantir a atuação dos agentes em sistemas marcados por incerteza genuína no sentido de Heiner.

O comportamento humano dirigido por objetivos ou motivações reflete simplesmente a forma do ambiente em que ele ocorre (Simon, 1999, pp. 53, 62). As características selecionadas do ambiente econômico capitalista devem refletir um determinado padrão de conduta competitiva, e este é o fator que motiva a tomada de decisão dos agentes em uma população relevante. Logo, estas são características coletivas que decorrem da interação entre os agentes. 


\section{RACIONALIDADE LIMITADA E O USO DE MODELOS DE SIMULAÇÃO BASEADOS EM AGENTES}

O instrumental de simulação em computador aplicado à economia é um campo de estudo bastante recente. Ainda que os primeiros trabalhos com aplicação deste instrumental nas ciências sociais sejam da década de 1960 (Axelrod, 2003, p. 21, nota 1 cita Cyert e March, 1963), as simulações passaram a ser cada vez mais utilizadas na década de 1990, ainda com certa resistência. Para Dweck (2006, p. 123, e 2010, p. 10), as críticas feitas ao uso de modelos de simulação partem tanto da ortodoxia quanto da heterodoxia. A principal delas é que a liberdade associada à técnica leva à falta de rigor na formulação do modelo e na interpretação dos resultados. Isto tende a promover o uso de modelos analíticos tradicionais que, por serem mais restritivos, impõem maior rigor à análise. No entanto, esta crítica é errônea, pois a liberdade dos modelos de simulação é fundamental para a compreensão de sistemas complexos, na medida em que torna o instrumental metodológico compatível com as características do objeto de análise, e não o contrário, como ocorre com os modelos tradicionais. Além disso, como explicam Borril e Tesfatsion (2012, p. 230), os modelos de simulação combinam elementos da matemática clássica com os da matemática construtiva. A matemática clássica com sua abordagem axiomática admite provas por contradição. A matemática construtiva, por outro lado, "exige uma prova direta da verdade de um enunciado, na forma de um procedimento computacional para eliminar tanto a falsidade quanto a indecidibilidade deste enunciado". A matemática construtiva interpreta a existência de uma entidade como a possibilidade de sua construção por procedimentos computacionais.

Ainda que toda teoria redefina seu objeto de análise, destacando os elementos considerados mais importantes e abstraia por simplificação aqueles menos relevantes ou recorrentes, é importante estabelecer limites à simplificação. Sobre o problema do uso de hipóteses simplificadoras nos modelos de simulação, Valente (1999a, p. 13) afirma: "Ao utilizar um modelo, não é mais necessário 'resolvê-lo'; é suficiente que se possa descrevê-lo. Isto significa que os elementos representados em um modelo (por exemplo, as firmas) não precisam ser definidos pelo resultado de seu comportamento subjacente (a determinação do preço que maximiza o lucro), mas podem ser representados pelo seu comportamento efetivo (por exemplo, a aplicação da regra de mark-up para determinar preço)".

As técnicas de simulação empregadas nos anos recentes têm sido desenvolvidas com base em duas abordagens distintas. A primeira tenta recriar a realidade, de forma que o modelo consiga reproduzir o maior número possível de características do ambiente representado, com toda a complexidade possível do mundo real. Este tipo de modelo tem grande aplicação no estudo de fenômenos relativos às ciências naturais e engenharia, pois os mecanismos causais dos fenômenos estudados nestas áreas são mais facilmente conhecidos. A segunda abordagem, que tem norteado a construção dos modelos aplicados aos fenômenos das ciências sociais, ao invés de tentar recriar a realidade, com o objetivo de prever o comportamento do sistema, 
procura examinar as relações entre os fatores que são condicionados pelos processos sociais (Brenner e Murmann, 2003), seguindo a lógica da racionalidade processual. A dificuldade de aplicar as técnicas de simulação às ciências sociais (com o propósito de fazer previsões sobre o comportamento futuro de um sistema) consiste no fato de que os mecanismos causais de tais sistemas, ou seja, as regras e rotinas que condicionam a interação social, mudam ao longo do tempo. Sendo assim, para que os resultados dos modelos de previsão sejam convincentes, seria necessário garantir que as regras e rotinas permanecessem inalteradas.

Um conjunto de modelos amplamente utilizado na ciência econômica é denominado "history-friendly" (Silverberg et al., 1988; Malerba e Orsenigo, 2002; Dosi et al., 2010). Ao contrário dos modelos de previsão, o conjunto "history-friendly" baseia-se nas relações causais extraídas da análise dos processos históricos dos fenômenos econômicos. É somente a partir da compreensão das relações causais fundamentais que se podem desenvolver tais modelos de simulação. O principal objetivo dos modelos de simulação "history-friendly" é compreender os processos determinados inteiramente pelas condições iniciais e parâmetros (as quais são denominadas, na literatura da economia evolucionista, path-dependency), independente das influências estocásticas.

Para Brenner e Murmann (2003), os modeladores da tradição history-friendly reconhecem o fato de que, mesmo compreendendo as relações fundamentais de causalidade dos fenômenos econômicos a partir da análise histórica da interação social, alguns resultados são fruto de influências estocásticas. Vale dizer, mesmo que o modelo seja fundamentado em parâmetros e condições iniciais determinados pela análise histórica dos fenômenos, as simulações podem apresentar resultados diferentes, ainda que as condições iniciais sejam as mesmas. O objetivo fundamental da simulação é conhecer os processos que se apresentam como regularidades, ou seja, características persistentes dependentes da trajetória do fenômeno estudado.

Como os processos estocásticos influenciam os resultados sociais, uma vantagem do uso de simulação é permitir a repetição do experimento simulado, usando as mesmas condições iniciais. Este exercício cria um grande número de observações que não estão disponíveis no registro histórico. Dessa forma, é possível analisar os resultados desses experimentos de simulação usando as ferramentas estatísticas tradicionais. Essa possibilidade viabiliza o desenvolvimento de análises contrafactuais. Segundo Brenner e Murmann (2003, p. 3), grande parte das explicações dos processos de interação social, ao menos implicitamente, contém a noção de que, se um determinado fator estivesse ausente, o resultado do processo social seria diferente. "Quando múltiplos agentes interagem em períodos mais longos, a articulação verbal sobre o que teria acontecido se certos fatores estivessem ausentes das condições iniciais pode facilmente ser errônea. Os cérebros humanos são excelentes para reconhecer padrões, mas são ruins para fazer inferências lógicas sequenciais."

Dada a racionalidade limitada dos agentes, traduzida nas limitações do cérebro humano para identificar as implicações causais de problemas que envolvem mais de uma variável ou múltiplas interações, o uso das técnicas de simulação permite 
obter os resultados de diferentes problemas de interação entre agentes em um ambiente complexo. Dessa forma, os modelos de simulação viabilizam a criação de informações sobre a relação entre duas ou mais variáveis. Quando diferentes cenários são modelados, o modelador consegue "repetir" a história no momento em que o modelo é rodado. Para criar diferentes cenários de simulação, o modelador necessita de profundo conhecimento do processo histórico que pretende investigar e de intuição. Os elementos que caracterizam cada cenário são formados pelo conjunto de parâmetros e condições iniciais do modelo, com base nos quais o modelo é calibrado para representar um ambiente específico.

Em alguns casos, os parâmetros podem ser observados com precisão, sendo possível fixá-los em um valor. Em outros casos, os dados disponíveis não são suficientes para determinar um valor exato para os parâmetros e, portanto, é necessário definir limites dentro dos quais eles podem variar seguindo determinada regra de comportamento. As regras e rotinas que condicionam o comportamento dos agentes devem ser fundamentadas em considerações teóricas e no comportamento passado do sistema que se pretende analisar, ou seja, em evidências empíricas.

Com relação à formulação dos pressupostos de um modelo de simulação, Brenner e Werker (2007) argumentam que se deve evitar que eles sejam puramente teóricos, tais como os modelos axiomáticos, bem como puramente empíricos. Se, por um lado, os pressupostos teóricos são construídos com base em algum conhecimento empírico (ao menos na forma de experiências observadas e conhecimento comum), por outro lado, pressupostos empíricos são formulados a partir de certas considerações teóricas básicas. Rodar a simulação significa obter conhecimento sobre as implicações dos supostos assumidos e, portanto, a essência da simulação é inferir implicações a partir de pressupostos. Segundo Axelrod (2003), não se deve confundir as análises feitas a partir de simulações com análises meramente dedutivas, pois ainda que os modelos de simulação sejam construídos a partir de um conjunto de hipóteses iniciais, os resultados não são utilizados para provar teoremas. Ao contrário, os dados gerados por simulação são obtidos com base em um conjunto de regras, rigorosamente especificadas, acerca do comportamento dos agentes, permitindo o desenvolvimento de trajetórias em aberto. A prova de um teorema envolve a descoberta de solução para um sistema cujas propriedades são previamente conhecidas. O instrumental de simulação, ao contrário, normalmente é utilizado para descobrir as propriedades do sistema.

Neste sentido, Brenner e Werker (2007, pp. 229-230) argumentam que o princípio de inferência utilizado na construção e análise de modelos de simulação aplicados à economia é a abdução (a partir de Lawson, 1997, p. 24). A aplicação deste princípio requer que informações detalhadas sobre um fenômeno sejam coletadas. Tais informações empíricas são utilizadas para encontrar diferentes padrões ou regularidades de comportamento, os quais são classificados a partir da identificação dos elementos estruturais subjacentes aos eventos e que os condicionam.

Brenner e Werker (2007, p. 235) identificam dois conjuntos de modelos claramente distintos. O primeiro conjunto é formado pelos modelos history-friendly e o segundo pelos modelos bayesianos. O conjunto history-friendly distingue-se do 
conjunto bayesiano por incorporar sistematicamente dados de estudos de caso como fonte de conhecimento sobre processos históricos reais para desenvolver modelos que sejam capazes de gerar processos com as mesmas características. Dessa forma, os parâmetros dos modelos e as relações básicas de causalidade entre as variáveis são obtidas pela análise histórica do fenômenos em observação, ou seja do estudo das trajetórias observadas no passado (path-dependency). No caso dos modelos bayesianos, dados empíricos são exaustivamente utilizados para definir as características básicas do modelo, pois os parâmetros, bem como as relações entre as variáveis do modelo, são muito pouco conhecidas previamente. Dessa forma, propriedades estatísticas e análise de sensibilidade em relação a efeitos aleatórios são amplamente utilizados para extrair dos dados empíricos um conjunto de parâmetros e condições iniciais capazes de calibrar o modelo. Em resumo, enquanto na abordagem history-friendly as informações sobre os processos que geram os fenômenos observados são estudadas, na abordagem bayesiana há muita informação sobre o fenômeno observado, mas pouquíssima informação acerca dos processos que geram o fenômeno.

Quanto à construção dos modelos de simulação, no que diz respeito ao grau de simplificação ou detalhamento das relações básicas entre as variáveis utilizadas para caracterizar um determinado fenômeno, algumas discussões (Edmonds e Moss, 2004; Brenner e Murmann, 2003; Pyka e Werker, 2009) giram em torno das vantagens relativas de modelos KISS ("keep it simple, stupid") ou KIDS ("keep it descriptive, stupid"). A escolha entre modelos mais simplificados e modelos mais descritivos é um problema recorrente em qualquer tipo de formalização. Silverberg (1997, p. 4) chama isso de "dilema do modelador": formular modelos que incorporem com maior riqueza de detalhes a complexidade do mundo real e, neste caso, correr o risco de ocultar propriedades fundamentais pelo excesso de complexidade incorporada ao modelo, ou formular modelos que reproduzam um problema altamente estilizado, que embora transparentes e passíveis de análise detalhada, serão sempre metáforas do mundo real.

Os modelos KISS procuram simplificar ao máximo as relações básicas, respeitando as características fundamentais do fenômeno investigado. Ainda que os pressupostos de um modelo de simulação possam ser bastante simples, os resultados observados são frequentemente complexos. Há muitos argumentos favoráveis à manutenção de um modelo simples, concernentes à facilidade para manipular, implementar e analisar os resultados. A abordagem KISS é baseada no princípio de que qualquer tentativa de representação do mundo real deve ser fundamentada nas características essenciais do fenômeno observado, removendo os elementos não fundamentais. A capacidade limitada dos seres humanos para processar informações e transformá-las em conhecimento explica a atração da maioria dos modeladores por modelos menos descritivos. O exemplo mais ilustrativo para justificar a importância de simplicidade na construção de modelos é pensar na inutilidade de um mapa em escala 1:1. Um exemplo adicional dessa abordagem é a afirmação de Axelrod (2003, p. 6), “[...] se uma simulação é utilizada para treinar a tripulação de um petroleiro, ou para desenvolver táticas para um novo caça 
aéreo, a precisão é importante e não a simplificação do modelo. Mas, se o objetivo é aprofundar ou compreender algum processo fundamental, a simplicidade dos pressupostos é importante e não a representação realística de todos os detalhes de um cenário particular”.

Contrariamente a esta abordagem, em defesa de modelos mais descritivos e menos simplificados, Edmond e Moss (2004) argumentam que o principal problema da abordagem KISS é pressupor que qualquer fenômeno possui características inerentemente simples, mesmo quando aparenta ser altamente complexo. Porém, o fato de que resultados complexos podem emergir de sistemas aparentemente simples nem sempre significa que o fenômeno possa ser reduzido a um modelo simples. Neste sentido, ignorar um aspecto relevante acerca do fenômeno investigado em benefício da simplicidade significa usar um modelo inadequado.

Muitas vezes, é possível construir modelos KIDS a partir de modelos KISS, pois conforme o modelo é construído, o modelador consegue obter maior conhecimento acerca do problema investigado, o que lhe permite descrever melhor o modelo. Para Valente (1999b, pp. 1-2), uma grande vantagem no uso de modelos de simulação em computador é que, frequentemente, antes de obter os resultados da simulação, o modelador já adquiriu um conhecimento profundo sobre o seu objeto de análise, pois para a construção do modelo faz-se necessária uma formalização detalhada da intuição geral que o modelador possui acerca das características básicas do objeto de estudo. Dessa forma, após a conclusão do processo inicial de formalização, o pesquisador possui um modelo de simulação representando sua intuição preliminar, ajustada pelos novos insights adquiridos ao longo do processo de construção do modelo.

Um importante tipo de simulação utilizado para estudar ambientes caracterizados pela presença de forte interação entre os agentes é a "agent-based modeling" (ABM). Os modelos baseados em agente utilizados nas ciências sociais têm como objetivo principal propiciar a compreensão de processos fundamentais presentes em uma variedade de aplicações, ao contrário de fornecer a representação acurada de uma aplicação empírica particular.

De acordo com Garson (2009, p. 271), a "simulação é uma forma de explorar os pressupostos, sem a preocupação de encontrar a solução 'correta' ou o conjunto ‘ótimo' de parâmetros”, em acordo com o suposto de racionalidade limitada dos agentes. Como nenhum modelo é capaz de representar de maneira acurada a complexidade incompreensível da realidade, as simulações na ciência econômica devem ser aplicadas de forma a simplificar a realidade permitindo que ela seja analisada e compreendida. Neste contexto, os modelos baseados em agente têm a vantagem de incorporar grande parte da complexidade do ambiente sem torná-la incompreensível, pois os elementos mais dinâmicos e de aparente desordem observados a partir da interação entre os agentes no âmbito micro resultam em propriedades emergentes e no surgimento de regularidades e auto-organização no âmbito macro, o que torna possível o surgimento de trajetórias inteligíveis em tempo discreto.

Para Dawid (2005), a emergência de regularidades macro baseadas em interações micro não coordenadas e descentralizadas é uma característica geral dos 
modelos baseados em agentes. Neste caso, a referência aos níveis micro e macro pode ser aplicada a qualquer tipo de modelagem envolvendo interação entre instâncias em diferentes níveis de agregação, como firmas dentro de uma indústria. Muitos modelos microeconômicos baseados em agente, os quais têm como ponto de partida o modelo de Nelson e Winter (1982), revelam que a heterogeneidade das estratégias de inovação não influencia somente o desenvolvimento das firmas individuais, mas tem efeitos sobre o desenvolvimento de toda a indústria. Nas palavras de Dawid (2005, p. 1241): "O uso de simulação em computador é uma característica marcante dos modelos baseados em agentes. A simulação permite vincular a interação entre as estratégias individuais de inovação, as estruturas de mercado e os efeitos micro ao desenvolvimento de variáveis industriais ou mesmo variáveis da economia como um todo, como a produtividade média dos fatores, $o$ número de firmas ou o crescimento econômico".

\section{CONSIDERAÇÕES FINAIS}

Este texto apresentou diversas questões epistemológicas, defendendo a superioridade dos modelos de simulação sobre os modelos da teoria econômica mainstream. Contudo, questões epistemológicas implicam (explícita ou implicitamente) concepções ontológicas distintas (Hoover, 1995; Lawson, 2003, cap. 1) e mesmo concepções matemáticas distintas (Borril e Tesfatsion, 2012, p. 230). O pressuposto de racionalidade está no núcleo duro de qualquer teoria que tem o objetivo de compreender a tomada de decisão dos agentes econômicos. Entre outros, os diversos pressupostos sobre a racionalidade dos agentes são um aspecto que divide teorias entre as da visão mainstream neoclássica e as alternativas. O pressuposto de que os agentes possuem racionalidade limitada está no núcleo duro da teoria evolucionista e da nova economia institucional. Contrariamente, assumir que a tomada de decisão dos agentes se fundamenta em racionalidade substantiva (inclusive pela suposição adicional de que todas as contingências futuras de uma determinada ação podem ser antecipadas) caracteriza as teorias que predizem resultados maximizadores, mesmo que tais resultados não sejam alcançáveis no mundo em que realmente vivemos.

As teorias que utilizam o suposto de racionalidade substantiva ou maximizadora estão inteiramente limitadas à ontologia de um sistema ergódico, segundo a qual a economia possui atratores que a conduzem para uma situação de equilíbrio. Todos os elementos que desestabilizam o sistema são considerados fatores exógenos, que não fazem parte da dinâmica do sistema (Vercelli, 1991, pp. 12-13). A teoria é desenvolvida com base em exercícios de estática comparativa, cuja importância reside no resultado final da ação - o equilíbrio - e não no processo gerador de (des) estabilidade. O comportamento dos agentes econômicos somente será maximizador se todos os possíveis resultados de suas ações forem passíveis de avaliação prévia. Ora, isto é impossível, dada a incerteza quanto aos estados futuros do mundo - resultantes da própria interação entre os agentes -, bem como suas limitações com- 
putacionais para processar todas as informações relevantes. Neste sentido amplo de "geração de variedades" causadoras de incerteza genuína, o modelo comportamental de racionalidade limitada é compatível com a abordagem evolucionista.

A superioridade das teorias baseadas em racionalidade limitada é, em grande medida, explicada pelo abandono de supostos relativos à ergodicidade e ao equilíbrio, não limitando suas conclusões à obtenção de resultados maximizadores e assumindo que o agente econômico faz o melhor da situação em que se encontra (ou seja, busca resultados meramente satisfatórios). De um lado, a admissão do comportamento satisfatório é o reconhecimento de uma limitação real dos agentes (Simon, 1999, p. 29). De outro lado, o abandono do suposto de maximização faz com que o interesse teórico se volte para a dinâmica do sistema, não apenas como um simples exercício de estática comparativa, mas como um exercício de compreensão dos mecanismos subjacentes promotores de heterogeneidade e mudança. Os mecanismos criados pelos agentes em interação para atenuar a incerteza genuína no sentido de Heiner, são os principais objetos de análise nessas teorias.

Neste sentido, o uso de modelos de simulação em computador constitui um instrumental adequado à análise de sistemas complexos, caracterizados pela não ergodicidade e pelo desenvolvimento de trajetórias em aberto. Ao contrário dos modelos analíticos tradicionais, o foco de modelos de simulação baseados em agentes $(\mathrm{ABM})$ não é o equilíbrio estático ou qualquer posição de steady state, mas sim a trajetória gerada e suas possíveis propriedades emergentes.

\section{REFERÊNCIAS BIBLIOGRÁFICAS}

Acha, V. e Cusmano, L. (2004) "Systems into Systems Run: complexity and evolutionary approaches to development". Texto apresentado na Conferência da European Association for Evolutionary Political Economy, Creta, 28-31 de outubro (mimeo).

Alchian, A. (1950) "Uncertainty, Evolution, and Economic Theory". The Journal of Political Economy, vol. 58 , no. 3, junho, pp. 211-221.

Axelrod, R. (2003) "Advancing the Art of Simulation in the Social Sciences". In: Conte, R.; Hegselmann, R. e Terna, P. (eds.) Simulating Social Phenomena. Berlim: Springer, pp. 21-40.

Azevedo, P.F. (1996) "Integração Vertical e Barganha". Tese (doutorado) - Faculdade de Economia e Administração, USP, São Paulo: mimeo.

Borril, P.L. e Tesfatsion, L. (2012) "Agent-Based Modeling: the right mathematics for the social sciences?”. In: Davis, J.B. e Hands, D.W. (eds.) The Elgar Companion to Recent Economic Methodology. Cheltenham: Edward Elgar, pp. 228-258.

Brenner, T. e J.P. Murmann (2003) "The Use of Simulations in Developing Robust Knowledge about Causal Processes: Methodological Considerations and an Application to Industrial Evolution”. Max Planck Institute, Papers on Economics \& Evolution, no. 0303, mimeo.

Brenner, T. e Werker, C. (2007) “A Taxonomy of Inference in Simulation Models". Computational Economics, vol. 30, no. 3, outubro, pp. 227-244.

Cyert, R. e March, J.G. (1963) A Behavioral Theory of the Firm. Englewood Cliffs, NJ: Prentice-Hall.

Conlisk, J. (1996) “Why Bounded Rationality”. Journal of Economic Literature, vol. 34, no. 2, junho, pp. 669-700.

Dawid, H. (2005) “Agent-Based Models of Innovation and Technological Change”. In: Tesfatsion, L. e Judd, K.L. (eds.) Handbook of Computational Economics. Vol. 2: Agent-Based Computational Economics. Amsterdã, North Holland, pp. 1235-1272. 
Dennett, D.C. (1998) A Perigosa Ideia de Darwin: a evolução e os significados da vida. Rio de Janeiro: Rocco.

Dequech, D. (1997) "Uncertainty in a Strong Sense: meaning and sources". Economic Issues, vol. 2, no. 2, setembro, pp. 21-43.

Dequech, D. (2001) “Bounded Rationality, Institutions and Uncertainty", Journal of Economic Issues, vol. 35, no. 4, dezembro, pp. 911-929.

Dosi, G.; Fagiolo, G.; Roventini, A (2010) "Schumpeter Meeting Keynes: a policy-friendly model of endogenous growth and business cycles". Journal of Economics Dynamics \& Control, vol. 34, no. 9, setembro, pp. 1748-1767.

Dunn, S.P. (2001) "Bounded Rationality, is not Fundamental Uncertainty: a Post Keynesian perspective”. Journal of Post Keynesian Economics, vol. 23, no. 4, verão, pp. 567-587.

Dweck, E. (2006) "Uma Análise da Interação Micro-Macro com Base em um Modelo Dinâmico Multissetorial de Simulação". Tese (doutorado) - Instituto de Economia, UFRJ, Rio de Janeiro: mimeo.

Dweck, E. (2010) “A Formalização da Interação Micro-Macrodinâmica: ABM e Simulações em Ciências Sociais”. Anais do XV Encontro Nacional de Economia Política, São Luis, 1-4 de junho.

Edmonds, B. e Moss, S. (2004) "From KISS to KIDS": an anti-simplistic modeling approach". In: Davidson, P.; Logan, B. e Takadama, K. (eds.) Multi-Agent and Multi-Agent Based Simulation. Nova York: Springer, pp. 130-144.

Fonseca, M.G.D. (2001) "Routines, Rules and Patters of Behaviour in the Context of Emergence". Texto apresentado na DRUID Nelson and Winter Conference. Aalborg, 12-15 de junho (mimeo).

Garson, G.D. (2009) “Computerized Simulation in the Social Sciences: a survey and evaluation”. Simulation and Gaming, vol. 40, no. 2, abril, pp. 267-279.

Gigerenzer, G. (2008) “Why Heuristics Work”. Perspectives on Psychological Science, vol. 3, no. 1, pp. 20-29.

Heiner, R.A. (1983) “The Origin of Predictable Behavior”. American Economic Review, vol. 73, no. 4, setembro, pp. 560-595.

Herscovici, A. (2004) “Irreversibilidade, Incerteza e Teoria Econômica: reflexões a respeito do indeterminismo metodológico e de suas aplicações nas ciências econômicas”. Estudos Econômicos, vol. 34, no. 4, outubro/dezembro, pp. 805-825.

Hoover, K.D. (1995) “Why does methodology matter for economics?” Economic Journal, vol. 105, no. 2, maio, pp. 715-734.

Koblitz, A. (2008) “Simon e Racionalidade Limitada”. Economia e Desenvolvimento, vol. 7, no. 2, julho/dezembro, pp. 251-285.

Lawson, T. (1997) Economics and Reality. Londres: Routledge.

Lawson, T. (2003) Reorienting Economics. Londres: Routledge.

Malerba, F. e Orsenigo, L. (2002) "Innovation and Market Structure in the Dynamics of Pharmaceutical Industry and Biotechnology: towards a history-friendly model”. Industrial and Corporate Change, vol. 11, no. 4, agosto, pp. 667-703.

Metcalfe, J.S.; Fonseca, M.G.D. e Ramlogam, R. (2002) "Innovation, Growth and Competition: evolving complexity or complex evolution”. Revista Brasileira de Inovação, vol. 1, no. 1, janeiro/julho, pp. $85-122$.

Nelson e Winter (1982) An Evolutionary Theory of Economic Change. Cambridge, MA: The Belknap Press.

Popper, K.R. (1978) Lógica das Ciências Sociais. Brasília: Editora da UnB, 1978.

Pyka, A. e Werker, C. (2009) "The Methodology of Simulation Models: chances and risks". Journal of Artificial Societies and Social Simulation (online), v. 12, n. 4, \$1.1-1.9.

Rizzello, S. (1999) The Economics of Mind. Aldershot: Edward Elgar.

Silverberg, G. (1997) "Review of Arthur, Durlauf and Lane (eds.) The Economy as an Evolving Complex System II”. [http://tuvalu.santafe.edu/ wbarthur/Reviews/Silverberg_Web.pdf].

Silverberg, G.; Dosi, G.; Orsenigo, L. (1988) "Innovation, Diversity and Diffusion: a self-organization model”. Economic Journal, vol. 38, no. 393, dezembro, pp. 1032-1054. 
Simon, H. A. (1955) “A Behavioral Model of Rational Choice”. Quartely Journal of Economics, vol. 69, no. 1, fevereiro, pp. 99-118.

Simon, H.A. (1983) Reason in Human Affairs. Stanford: Stanford University Press.

Simon, H.A. (1986) "Rationality in Psychology and Economics". Journal of Business, vol. 59, no. 4, parte 2, outubro, pp. S209-S224.

Simon, H.A. (1999) The Sciences of the Artificial, 3a ed. Cambridge, MA: The MIT Press.

Sudgen, R. (1991) "Rational Choice: A Survey of Contributions from Economics and Philosophy". The Economic Journal, vol. 101, no. 407, julho, pp. 751-785.

Tisdell, C.A. (1996) Bounded Rationality and Economic Evolution: a contribution to decision-making, economics and management. Aldershot: Edward Elgar.

Valente, M. (1999a) "Evolutionary Economics and Computer Simulations - A Model for the Evolution of Markets. Vol. I: Consumer Behaviour and Technological Complexity in the Evolution of Markets”. PhD Thesis, Department of Business Studies, University of Aalborg, outubro, mimeo.

Valente, M. (1999b) "Evolutionary Economics and Computer Simulations - A Model for the Evolution of Markets. Vol. II: Laboratory for Simulation Development - A Proposal for Simulation Models in Social Sciences". PhD Thesis, Department of Business Studies, University of Aalborg, outubro, mimeo.

Vercelli, A. (1991) Methodological Foundations of Macroeconomics: Keynes and Lucas. Cambridge: Cambridge University Press.

Vromen, J. (2004) "Conjectural Revisionary Economic Ontology: outline of an ambitious research agenda for evolutionary economics”. Journal of Economic Methodology, vol. 11, no. 2, junho, pp. 213-247. 